\title{
ON THE POWER OF PLANNED INFECTIONS IN NETWORKS
}

\author{
Michael Brautbar, ${ }^{1}$ Moez Draief ${ }^{\mathbf{2}}$, and Sanjeev Khanna ${ }^{\mathbf{3}}$ \\ ${ }^{I}$ Electrical Engineering and Computer Science, Massachusetts Institute of \\ Technology, Cambridge, Massachusetts, USA \\ ${ }^{2}$ Electrical and Electronic Engineering, Imperial College London, London, UK \\ ${ }^{3}$ Computer and Information Science, University of Pennsylvania, Philadelphia, \\ Pennsylvania, USA
}

\begin{abstract}
Over the last decade we have witnessed the rapid proliferation of online networks and Internet activity. Although such activity is generally considered a blessing, it also brings with it a large increase in risk of computer malware-malignant software that actively spreads from one computer to another. To date, the majority of existing models of malware spread use stochastic behavior, when the set of neighbors infected from the current set of infected nodes is chosen obliviously. In this work, we initiate the study of planned-infection strategies that can decide intelligently which neighbors of infected nodes to infect next in order to maximize their spread, while maintaining a "signature" similar to the oblivious stochastic infection strategy in order not to be discovered. We first establish that computing optimal and near-optimal planned strategies is computationally hard. We then identify necessary and sufficient conditions in terms of network structure and edge infection probabilities such that the planned process can infect polynomially more nodes than the stochastic process while maintaining a similar "signature" as the oblivious stochastic infection strategy. Among our results is a surprising connection between an additional structural quantity of interest in a network, the network toughness, and planned infections. Based on the network toughness, we characterize networks where existence of planned strategies that are pandemic (infect all nodes) is guaranteed, as well as efficiently computable.
\end{abstract}

\section{INTRODUCTION}

Over the last decade we have witnessed the rapid proliferation of online networks and Internet activity. Although such a proliferation is considered by many a blessing, it brings with it an increase in risk of computer malware-malignant software that actively spreads from one computer to another. Indeed, a long thread of research has been devoted to understanding the spread of malicious malware such as computer viruses, computer worms, and other malignant forms of computer infection; cf. [3, 17, 18, 20,22]. However, such work has, so far, only considered only oblivious malware propagation, by which the spreading malware does not behave strategically in its choice of which nodes to spread to. What is now a standard way of containing the spread of malware is the control of the amount of

Address correspondence to Michael Brautbar, Laboratory for Information and Decision Systems, Electrical Engineering and Computer Science, Massachusetts Institute of Technology, 77 Massachusetts Avenue, Cambridge, MA 02139, USA. E-mail: mbrautbar@gmail.com

Color versions of one or more of the figures in the article can be found online at www.tandfonline.com/uinm. 
information that spreads from one computer to others [3,20]. This is also known as throttling. Under rate control, a malware that does not want to get detected spreads obliviously to a small set of neighbors while abiding by the rate constraint. Inspired by this fact, in this work we initiate the study of intelligent malware propagation, whereby the spreading malware can strategically decide which neighboring nodes to infect under rate constraints in order to maximize the total number of infections over time. Each edge $(u, v)$ is equipped with an edge weight $p(u, v)$ representing the amount of a typical and normal communication between the two nodes $u$ and $v$. Typical examples of such networks include e-mail networks, instant messaging networks, and online social networks, among others. Under the rate constraint, a malware spreading from an infected node $u$ can infect at most a number of nodes that is not more than the typical "signature" of communication, namely,

$$
\left\lceil\sum_{\{v \text { neighbor of } u\}} p(u, v)\right\rceil .
$$

More generally, we will demand that for any subset $X$, the malware must not infect more nodes than the amount of traffic involving $X$ permits it to, namely,

$$
\left\lceil\sum_{u \in X} \sum_{\{v \text { neighbor of } u\}} p(u, v)\right\rceil .
$$

In particular, such malware can use the structure of the network in order to choose which of the neighboring computers to infect from a newly infected node. We initiate a detailed comparison of the behavior of a planned infection, which can use the network structure, to that of an oblivious stochastic infection, that spreads according to the standard Independent Cascades diffusion model. In order to defend against future malware, it is of primary importance to first analyze the planned-infections strategy and to contrast its behavior with that of the oblivious stochastic strategy.

We would like to further emphasize the need to understand the behavior of infections with the following example, comparing the behavior of a well-planned infection to that of a simple heuristic.

Example 1.1. Consider a path connected at one of its ends to the root of a two-level binary tree. See Figure 1 for an illustration. Set all edge weights to $1 / 2$. A greedy strategy may consist, for each newly infected node $u$, of a plan to infect its $r_{u}$ neighbors of highest degree, where $r_{u}$ is the rate constraint of $u$. However, such a strategy would miss the path altogether. In contrast, a planned strategy with a global knowledge of the graph can plan ahead and infect the whole path, by starting from the root of the tree but spending its budget to infect the next node on the path (and the extra budget to infect part of tree).

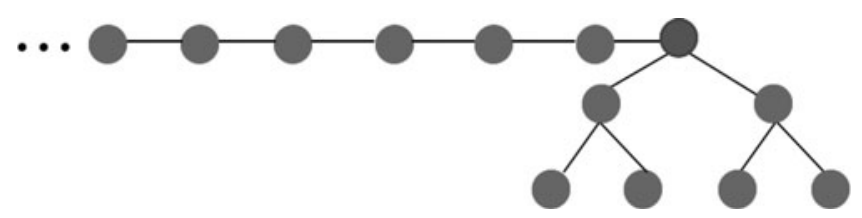

Figure 1 A path connected to a two-level binary tree. 
Whereas a greedy strategy would infect only $O(1)$ nodes, a well-planned strategy would infect $n-O(1)$ nodes.

\subsection{Our Results}

We first show that the problems of computing optimal and near-optimal planned strategies under typical constraints are computationally hard. We then identify necessary and sufficient conditions in terms of network structure and edge infection probabilities such that the planned-infection process can infect polynomially more nodes than the stochastic process while maintaining a similar "signature" as that of the oblivious stochastic infection strategy. Our first set of results show that when the minimum weighted graph cut value is $\Omega(\log n)$ (on a network with $n$ nodes), the standard oblivious stochastic infection strategy can essentially infect all nodes. Thus, the interesting regime to analyze is when the minimum weighted cut value is $o(\log n)$. In this regime, we demonstrate that optimal planned infection can be pandemic (namely infect all nodes) whereas the oblivious stochastic strategy infects, in expectation, a constant number of nodes. We then identify a surprising connection between an additional structural quantity of interest in a network, the network toughness, and planned infections. Based on the network toughness, we characterize conditions guaranteeing the existence of a pandemic planned strategy as well as its efficient computation.

\subsection{Related Work}

Most work on computer malware has been focused on virus and worm propagation $[18,20]$. The vast majority of the literature has focused on modeling and simulations of the behavior of a stochastic malware, which spreads according to the Independent Cascades model or its extension to repeated infection attempts, the Susceptible-Infected model [8, 20-23]. However, none of these studies consider intelligently designed malware that can choose which nodes to infect based on some prior computation or knowledge of the network.

Another line of research that we would like to mention is that devoted to error and attack tolerance in online networks; see the seminal work of [1] and its long line of followup research such as [10]. The main thread of research is devoted to understanding how attacking and removing, in an unweighted graph, nodes of high-degree results in more parts of the network becoming disconnected than attacking and removing the same number of nodes, obliviously at random. In contrast to this work, we are interested in analyzing cascading effects that spread through the network, rather than a single attack and removal of nodes (and edges). Furthermore, our main interest is in coordinated attacks that are strategically designed, and so the type of node first targeted is chosen in order to maximize a global effect in a provable way (resulting in infecting as many nodes as possible) rather than being based on local heuristics (choosing high degree nodes).

\subsection{Outline}

In Section 2 we provide a detailed definition of what a planned infection comprises and the description of the behavior of the oblivious stochastic infection strategy. In Section 3 we discuss the computational complexity of computing optimal, as well as near optimal, planned-infection strategies. In Section 4 we provide necessary conditions in terms of the network cuts such that the planned process can infect polynomially more nodes than the stochastic process. In Section 5 we provide necessary and sufficient conditions for 
the existence of a pandemic planned-infection strategy and provide efficient algorithms for computing such a strategy. Finally, in Section 6 we summarize our contributions and list several intriguing directions for future research.

\section{MODEL AND PRELIMINARIES}

The Input Network. We are given an undirected, edge-weighted network $G=(V, E, p)$ on $|V|=n$ nodes, $|E|=m$ edges and an edge weight function $p: E \rightarrow(0,1]$. We think of the weight $p(u, v)$ as the average amount of communication between neighbors $u$ and $v$ over a typical period of time. We will be particularly interested in the behavior of planned infections and the independent cascade model on the input network.

Planned Infections. A planned strategy $A$ is a rooted tree $T_{A}$, rooted at its seed node of choice (namely, the source of infection), which spans some arbitrary subset $S$ of nodes. Each node $u$ in $T_{A}$ is responsible for infecting its children in $T_{A}$. For any subset $X$ of nodes in $T_{A}$, let

$$
\operatorname{infect}(A, X)=\left\{v \in T_{A}: v \text { 's parent in } T_{A} \text { belongs to } X\right\} \text {. }
$$

We say that a planned strategy $A$ (with its rooted tree $T_{A}$ ) infects a set $S$ of nodes, while obeying the first order constraints, if its rooted tree $T_{A}$ spans $S$ such that for every subset $X \subseteq S$ of nodes we have,

$$
|\operatorname{infect}(A, X)| \leq\left\lceil\sum_{u \in X} \sum_{v:(u, v) \in E} p(u, v)\right\rceil .
$$

Namely, the number of node infections attributed to $X$ is constrained by the ceiling of the total weight adjacent to the set $X$.

In this article we consider only planned infections that obey the first-order constraints; unless stated explicitly otherwise, a planned strategy would be assumed to obey the firstorder constraints. We will call the problem of finding a first-order constrained planned infection that maximizes the number of infections the planned-infection maximization problem. ${ }^{1}$ A planned strategy will be called pandemic if it infects all nodes.

Independent Cascades Infection Model. The Independent Cascades (IC) model of infection was formalized by Kempe et al. [14] and is by now a standard model to describe infection propagation in social and other contact networks [11]. The IC model can be thought of as a discrete version of the known Susceptible-Infected-Removed (SIR) model. The IC diffusion spreads via a random process, beginning at its seed node of choice from $V$. The process proceeds in rounds. In each round, a node $u$ that got infected in the previous round gets a chance to subsequently infect each healthy neighbor $v$, with probability equal to the weight of the edge $(u, v)$, namely $p(u, v)$. The node $u$ becomes then recovered and does not spread the virus any further. If multiple nodes try to infect a new node in the current round, then each succeeds, independently, according to the corresponding edge weight. In this article, we will focus on the IC process only on undirected graphs. Throughout the text, we shall also refer to the stochastic infection strategy, as defined by the IC process,

\footnotetext{
${ }^{1}$ It is not difficult to see that there exists a deterministic optimal planned infection: any stochastic infection is a convex combination of trees, so one can take the best tree without loss of generality.
} 
as the oblivious stochastic strategy or sometimes as the stochastic infection or stochastic diffusion.

Quantities of Interest. All graphs considered in the article are undirected graphs. Throughout our analysis, we will frequently refer to the minimum weighted cut value and maximum weighted cut value in the weighted input graph: the minimum (resp. maximum) weighted cut value, denoted $\Phi_{G}^{\min }\left(\operatorname{resp} . \Phi_{G}^{\max }\right)$, is the value of the cut $(S, \bar{S})$ that minimizes (resp. maximizes) the sum

$$
\Phi_{G}(S):=\sum_{\{u \in S, v \in \bar{S},(u, v) \in E\}} p(u, v) .
$$

When the identity of $G$ is clear, we will often omit the subscript $G$.

Notation. We denote by $|S|$ the size of a set $S$. We denote the degree of a node $u$ in a graph $G$ by $d_{G}(u)$. We shall call a graph traceable if it has an Hamiltonian path. All logarithms in the paper are in base 2 .

\section{HARDNESS OF MAXIMIZING PLANNED INFECTIONS}

In this section, we show that in general, the planned-infection problem is difficult to approximate under first order constraints.

Definition 3.1. Let $P$ be a discrete maximization problem, and let $P(x)$ denote its optimal value on an input instance $x$. For any $c \geq 1$, an algorithm $A$ is a c-approximation algorithm for $P$ iffor any input $x$, the algorithm $A$ returns a value in the interval $[P(x) / c, P(x)]$.

Definition 3.2. A discrete maximization problem $P$ is called $c$-hard to approximate under some complexity-theoretic assumption $\mathcal{A}$ if existence of a polynomial-time c-approximation algorithm for $P$ will refute the assumption $\mathcal{A}$.

Theorem 3.3. For any $0<\epsilon<1$, the planned-infection maximization problem is $2^{\left(\log ^{1-\epsilon} n\right)}$-hard to approximate, unless $\boldsymbol{N P} \subseteq \operatorname{DTIME}\left(2^{O\left(\log ^{1 / \epsilon} n\right)}\right)$.

Proof. The proof is by reduction from the longest path problem on undirected graphs. Let $G(V, E)$ be the input graph for the longest path problem. We create an instance of the planned-infection problem from $G$ as follows. Starting with the graph $G$, we attach $\left(n-d_{G}(u)\right)$ auxiliary vertices to each vertex $u \in V$. Assign a weight of $1 / n$ to each edge in the resulting graph. Let $H$ be the resulting graph.

Note that any planned-infection strategy in $H$ obeying the first-order constraints must be a path, since the total incident weight on any node in $H$ is at most 1 (and is exactly 1 for nodes from $G$ ). If the path length is $\ell$ in $H$, it translates to a path of length at least $\ell-2$ in $G$ (which is at least $\ell / 2$ for $\ell \geq 4$ ), and a path of length $\ell$ in $G$ translates to an infection strategy following a path of length $\ell$ in $H$. The longest path problem is $2^{\left(\log ^{1-\epsilon} n\right)}$-hard to approximate in undirected graphs, unless NP $\subseteq$ DTIME $\left(2^{O\left(\log ^{1 / \epsilon} n\right)}\right)[13]$, and the result easily follows.

We next show that the problem remains difficult to approximate to within any constant factor even when the input instances are restricted to regular graphs with uniform infection probabilities. 
Theorem 3.4. The planned-infection maximization problem does not admit a constant factor approximation in undirected regular graphs with uniform edge weights, unless $\boldsymbol{P}=\boldsymbol{N P}$.

Proof. Let $G$ be an undirected $k$-regular graph, $k \geq 2$ with uniform infection probability of $1 / k$ on edges. Now, the problem of finding a good strategy obeying first-order constraints becomes exactly the problem of finding a long path in the graph. Thus the problem of planned-infection maximization is as difficult to approximate as the longest path on regular graphs. Even in 3-regular Hamiltonian graphs, the longest-path problem is known not to have any constant factor approximation, unless $\mathbf{P}=\mathbf{N P}[6]$

\section{A CUT-BASED ANALYSIS}

We next proceed to exploring networks in which a planned infection can infect polynomially more nodes than the oblivious stochastic strategy. We will show that two important parameters in understanding this goal are the value of the minimum weighted cut, $\Phi_{G}^{\min }$, and the value of maximum weighted cut, $\Phi_{G}^{\max }$, in the input graph $G$.

We first show that if $\Phi_{G}^{\mathrm{min}}$ is at least logarithmically large, the oblivious stochastic strategy is essentially pandemic.

Theorem 4.1 (Theorem 1 of [2]). Let $G=(V, E, p)$. For every positive constant $b$, there exists a constant $c=c(b)>0$ so that if $\Phi_{G}^{\min } \geq c \log (n)$, then the probability that a realization of $G$ is disconnected, where each edge $(u, v) \in E$ is kept with probability $p(u, v)$, is at most $1 / n^{b}$.

By the theorem we conclude,

Corollary 4.2. For c large enough, the oblivious stochastic strategy would infect at least $n-o(1)$ nodes in expectation.

Thus, in this parameter regime, an improvement using planned-infection strategies would be nonsignificant over the oblivious stochastic strategy. We note, however, that having a logarithmically large minimum cut is a quite stringent condition; in particular, any graph that has even one node with degree of size $o(\log (n))$ would violate the minimum cut condition. It is, thus, of high interest to analyze the regimes when this condition is violated. For this purpose, we next show that no planned strategy can infect more than $\Theta\left(\Phi_{G}^{\max }\right)$ nodes, and that when $\Phi_{G}^{\max }$ is large enough, a polynomial gap between an optimal planned-infection strategy and the oblivious stochastic strategy is feasible.

Theorem 4.3. For any graph $G$, no planned-infection strategy obeying first-order constraints, as well as the oblivious stochastic strategy, can infect more than $\Theta\left(\Phi_{G}^{\max }\right)$ nodes.

However, for any value $n$ there exists a graph $G$ on $n$ nodes with $\Phi_{G}^{\max }=\Theta(n)$ such that an optimal planned strategy obeying first order constraints infects $\Theta\left(\Phi_{G}^{\max }\right)$ nodes whereas the oblivious stochastic strategy infects only $O(1)$ nodes in expectation. Moreover, the gap result holds on graphs in which the edge infection probability is uniform and a constant (independent of $n$ ). 
Proof. To prove the first part, we make use of the following known fact that can be easily proven using the probabilistic method.

Fact 4.4. For any weighted undirected graph $G=(V, E, p)$,

$$
\Phi_{G}^{\max } \geq 1 / 2 \sum_{(u, v) \in E} p(u, v) .
$$

Now consider any planned-infection strategy $A$, and let $T_{A}$ be its tree of infections; let $S$ be the set of nodes infected. Ignoring the root of $T_{A}$, either the total number of nodes at the odd levels of $T_{A}$ must be at least $(|S|-1) / 2$ or the total number of nodes at the even levels of $T_{A}$ must be at least $(|S|-1) / 2$. Since all infections at odd levels have to be attributed to nodes at the even levels (and vice versa), if the tree $T_{A}$ conforms to first-order constraints, then we must have

$$
(|S|-1) / 2 \leq\left\lceil 2 \Phi_{G}^{\max }\right\rceil .
$$

Because this holds for any choice of tree $T_{A}$ (and, hence, any planned strategy), any plannedinfection strategy infects at most $\Theta\left(\Phi_{G}^{\max }\right)$ nodes. A simple argument shows the same result for the oblivious stochastic process: any node $u$ can infect at most $\sum_{v:(u, v) \in E} p(u, v)$ new nodes in expectation, and thus, the total number of infections is, in expectation, at most

$$
\sum_{u \in V} \sum_{v:(u, v) \in E} p(u, v)=\Theta\left(\Phi_{G}^{\max }\right) .
$$

We now prove the other part of the theorem. To show this we need to provide a graph $G$ on $n$ nodes such that an optimal planned-infection strategy can infect $\Phi_{G}^{\max }=\Theta(n)$ nodes while the oblivious stochastic strategy infects $O(1)$ in expectation.

For simplicity of exposition, assume that $n$ is even. Take a cycle on $n / 2$ nodes and set each edge probability on the cycle to be $2 / 3$. Now attach to each node $u_{i}$ on the cycle an auxiliary node $v_{i}$ using also an edge of probability $2 / 3$. See Figure 2 for an illustration.

Note that by Fact 4.4, the value of the maximum weighted cut $\Phi_{G}^{\max }$ is $\Omega(n)$. Clearly, $\Phi_{G}^{\max } \leq|E|=n$ and so $\Phi_{G}^{\max }=\Theta(n)$, as required.

A planned-infection strategy can infect all nodes by following any rooted spanning tree of the graph. This strategy satisfies first-order constraints because each node $u_{i}$ has an infection budget of exactly 2 , and this strategy needs to infect on each step at most two new nodes.

However, for any choice of the seed vertex, a stochastic strategy obtains only $O(1)$ nodes in expectation. This holds even though a newly infected "cycle" node infects in expectation $4 / 3>1$ of its neighbors. To see this, note that the infection survives for $k$ steps on the cycle with probability at most $2\left(\frac{2}{3}\right)^{k}$ and so it can infect, in expectation, at most $\sum_{k} 4 k\left(\frac{2}{3}\right)^{k}=12 \sum_{k} k\left(\frac{2}{3}\right)^{k} \frac{1}{3}=12 \cdot \frac{2}{3} / \frac{1}{3}=24=O(1)$ nodes.

\section{PANDEMIC INFECTIONS}

In this section, we further explore the setting where the value of the minimum weighted cut is $o(\log n)$. As we have seen earlier, in this setting the gap between a planned infection 


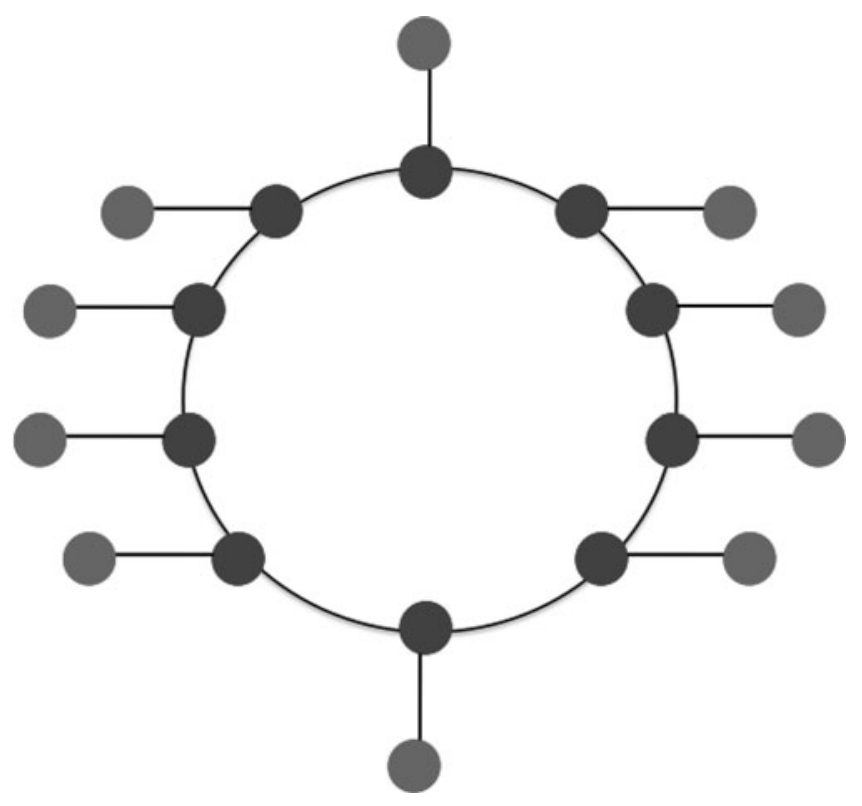

Figure 2 An example illustrating the construction.

(obeying first-order constraints) and oblivious stochastic diffusion can be as large as $\Omega(n)$. We obtain here sufficient and necessary conditions for a planned infection to become pandemic (i.e., infect all nodes) by relating existence of such strategies to the notion of toughness of the graph.

The notion of graph toughness was first introduced in order to study conditions for the existence of Hamiltonian cycles in graphs, see [4]. Given an undirected graph $G=(V, E)$ and a subset of nodes $S$, let $|S|$ be the size of $S$ and $c_{G}(S)$ be the number of connected components in the graph induced on $V \backslash S$ obtained from $G$ by deleting all nodes in the set $S$.

Definition 5.1. The toughness of the graph $G$, where $G$ is not the complete graph, denoted by $\tau(G)$ is defined as follows

$$
\tau(G)=\min _{S \subset V, c_{G}(S)>1} \frac{|S|}{c_{G}(S)} .
$$

The toughness of the complete graph is defined to be $\tau(G)=\infty$.

Toughness of a cycle, on the other hand, is 1 because, by deleting any subset of $k$ nodes, we can create at most $k$ connected components, and removing two nodes with no edge between them creates exactly two components. As another example, it is easy to verify that the toughness of a tree with maximum degree $\Delta$ (and at least three nodes) is $1 / \Delta$. It is also easy to verify that the toughness of a graph is positive if and only if the graph is connected.

There is a vast literature on the connection between graph toughness and spanning trees; see [16] for a recent survey. In what follows, we will show a close connection between existence and algorithms for planned infections that are pandemic, and the toughness of the underlying connection graph. 
We start by developing sufficient conditions under which there exists a spanning tree that can be exploited by a planned strategy obeying the first-order constraints.

Theorem 5.2 (Pandemic Theorem). For any connected, weighted undirected graph $G=$ $(V, E, p)$ such that

$$
\forall u \in V, \sum_{v:(u, v) \in E} p(u, v) \geq\left\lceil\frac{1}{\tau(G)}+1\right\rceil,
$$

there is a planned strategy that infects all nodes in $G$ and obeys the first-order constraints. Moreover, if

$$
\forall u \in V, \sum_{v:(u, v) \in E} p(u, v) \geq\left\lceil\frac{1}{\tau(G)}+2\right\rceil,
$$

then there is a polynomial-time computable planned strategy that infects all nodes in $G$ and obeys the first-order constraints.

Proof. By Win's Theorem [19], every undirected graph $G$ with toughness $\tau(G)$ has a spanning tree with maximum degree bounded by $d=\lceil(1 / \tau(G)+2)\rceil$. Let $T$ be such a tree. Root this tree at a leaf node $s$ and let this node be the seed. The planned strategy is to infect all nodes of $G$, starting with node $s$, by using the edges of $T$. Now, no node is responsible for infecting more than $d-1$ children, and so the first-order constraints are obeyed: for each node $u$, its degree minus one is at most $\lceil(1 / \tau(G)+2)\rceil-1=\lceil(1 / \tau(G)+1)\rceil \leq$ $\sum_{v:(u, v) \in E} p(u, v)$. To show the second part of the theorem, we make use of an algorithmic result by Fürer and Raghavachari [12], which states that if there exists a spanning tree of degree at most $\Delta$, then one can construct in polynomial time a spanning tree of degree at most $\Delta+1$. The assertion of the theorem then follows similarly to the previous case, where now for each node $u,\lceil 1 / \tau(G)+2\rceil \leq \sum_{v:(u, v) \in E} p(u, v)$.

\subsection{Applications of the Pandemic Theorem}

We next provide several examples demonstrating the effectiveness of the Pandemic Theorem.

Example 5.3. A graph is called claw free if it does not have $K_{1,3}$ as an induced subgraph (namely, a "star" graph with three leaves). Take any 2-node connected claw-free graph. As shown in [15], any 2-node connected claw-free graph has toughness at least one. Thus, if for each node in such a graph the total weight on the edges adjacent to it is at least 2, then by the Pandemic Theorem there is a planned strategy that can actually infect all nodes.

Example 5.4. In their seminal paper, Bauer et al. [5] refuted Chvátal's conjecture on the connection between graph toughness and Hamiltonian graphs by constructing a nonHamiltonian graph with toughness at least 2. To show this, Bauer et al. developed a method that produces infinitely many nontraceable graphs with toughness at least 2 and all nodedegrees as large as we want (a graph is nontraceable if it does not contain a Hamiltonian path). Their method takes any nontraceable graph as input and uses it as a gadget to create the final graph. We will skip over the details of the construction because they are highly technical; the interested reader is referred to the complete description of the method in 
Section 2 of [5]. As a corollary we can conclude that for any such graph, if for each node the total weight on the edges adjacent to it is at least 2, then by the Pandemic Theorem there is a planned strategy that infects all nodes.

Example 5.5. We next illustrate the power of the second part of the Pandemic Theorem. Let $r \geq 3$ be integral and $\mathcal{G}_{r}$ be the family of Hamiltonian graphs with maximum degree $r$ where each edge has weight $1 / r$. It has been shown [6] that in 3-regular Hamiltonian graphs, the longest-path problem is not constant factor approximable, unless $\mathbf{P}=\mathbf{N P}$ [6]. One can easily extend this result to Hamiltonian graphs with maximum degree $r$ (see Lemma A.1 in the appendix for details). Because a graph in $\mathcal{G}_{r}$ is Hamiltonian, its toughness is at least 1. Thus, although the longest simple path equals the number of nodes in the network minus one, any polynomial-time computable planned strategy that follows a path can only ensure infecting a diminishing fraction of the nodes for graphs in $\mathcal{G}_{r}$ (unless $\mathbf{P}=\mathbf{N P}$ ). However, by the second part of the Pandemic Theorem, one can construct in polynomial-time a planned strategy that infects all nodes for any graph in $\mathcal{G}_{r}$. Such a strategy will be a spanning tree of maximum degree 3 .

\subsection{Tightness of the Pandemic Theorem}

We next show that if we slightly weaken condition (5.2), stated in Theorem 5, the result no longer holds. Specifically, if we require only that

$$
\forall u \in V, \sum_{v:(u, v) \in E} p(u, v) \leq\left\lfloor\frac{1}{\tau(G)}+1\right\rfloor,
$$

then there exist infinitely many networks such that an optimal planned strategy, obeying first-order constraints, is not pandemic (infects all nodes). To see this, consider any graph with toughness $\tau(G)>1$ that does not have a Hamiltonian path. We know of the existence of such graphs from the seminal work of [5]. As $\left\lfloor\frac{1}{\tau(G)}+1\right\rfloor=1$ when $\tau(G)>1$, any pandemic planned infection must be a Hamiltonian path, which does not exist in the graph.

In particular, the above discussion provides a necessary condition for pandemic infections on nontraceable graphs.

Corollary 5.6. Let $G$ be a nontraceable graph with toughness $\tau(G)>1$. If

$$
\forall u \in V, \quad \sum_{v:(u, v) \in E p(u, v)} \leq\left\lfloor\frac{1}{\tau(G)}+1\right\rfloor,
$$

then any planned strategy, obeying first-order constraints, is not pandemic (infects all nodes).

One can also infer a tighter condition for graphs with toughness smaller than 1 . We show that if we allow just 3 nodes to violate condition (5.2) by only a slack of 2 , that is, allow 3 nodes $u$ with $\sum_{v:(u, v) \in E} p(u, v)=\lceil(1 / \tau(G)-1\rceil$, then, for infinitely many networks, an optimal planned strategy is not pandemic (infects all nodes). To see this, let $k \geq 2$ be an integer and consider the following family of graphs $H_{n, k}$, where $n \geq 3 k(k+4)+3$ is integral. Take three nodes $v_{1}, v_{2}, v_{3}$, and connect them in a triangle. In addition, create $3 k$ 


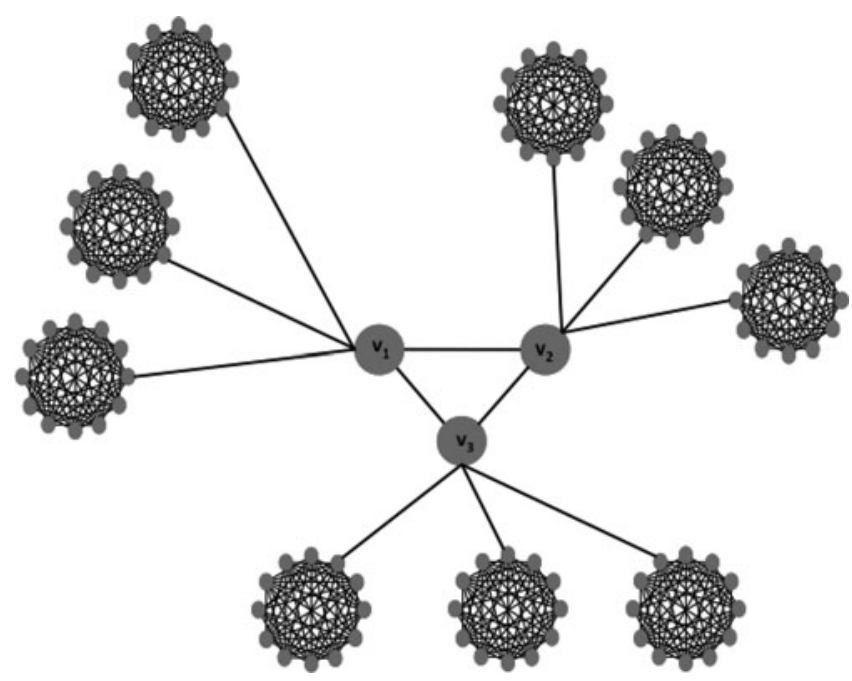

Figure 3 An example illustrating the construction for $k=3$.

vertex-isolated cliques, indexed $C(i, j)$ for $1 \leq i \leq 3,1 \leq j \leq k$, each on $(n-3) / 3 k$ nodes. To complete the construction, connect each node $v_{i}$ to some node in each clique $C(i, j)$ ("a representative"), where $1 \leq j \leq k$. Thus, the degree of each node $v_{i}$ in the construction is $k+2$; in addition to being connected to $k$ representatives it is also connected to the other $v_{i}$ nodes. Set all edge weights to $1-1 / k$, except for each $i$ of the edges connecting $v_{i}$ to the cliques $C(i, 1)$ and $C(i, 2)$; the weight on each of these edges is set to $1 / 2$. See Figure 3 for an illustration of this construction for $k=3$.

We now observe a few simple facts about the graph $H_{n, k}$. First, its toughness is $1 /(k+1)$. (The toughness value is achieved by removing one node $v_{i}$ from the graph; removal of any other set of nodes that disconnects the graph will give a value larger than $1 /(k+1)$ for the ratio of removed nodes to the number of connected components created.) Second, it has a spanning tree (tree spanning all graph nodes). Third, in each of its spanning trees, each $v_{i}$ must be connected to a representative from each $C(i, j)$ for $1 \leq j \leq k$. In addition, one of the $v_{i}$ s must be connected to the two other $v_{i}$ s (otherwise, the spanning tree is disconnected), and so its degree in the spanning tree must be $k+2$. Last, except for the three $v_{i} \mathrm{~s}$, the sum of edge weights touching any node is at least $k+2$ (as $\frac{n-3}{3 k} \geq k+4$ condition (5.2) holds for all clique nodes). However, the sum of edge weights touching a $v_{i}$, namely $\sum_{u:\left(v_{i}, u\right) \in E} p\left(v_{i}, u\right)$, is $k(1-1 / k)+1 / 2 \cdot 2=k$. Thus, the degree of each of the $v_{i}$ s in a tree representing a planned infection can be at most $k+1$, which is strictly smaller than $k+2$, and so any such infection cannot infect all nodes in the graph. In fact, a constant fraction of the graph nodes will not be infected-all the nodes belonging to one of the cliques $C(i, j)$.

\subsection{Toughness and Stochastic Diffusion}

In this section we show that for a given value of the toughness $\tau$, there exist infinitely many graphs such that the stochastic diffusion can infect only $O(\tau)$ nodes, for $\tau \geq 4$. In light of Theorem 5, on such graphs the gap between the stochastic diffusion and the planned diffusion is large. 
Theorem 5.4. For any value of the toughness $\tau \geq 4$ and positive integer $\ell \geq 3$, there exists a weighted undirected graph $G=(V, E, p)$ of $n=\tau \ell$ nodes and toughness $\tau$, such that condition (5.2) is satisfied (hence, the planned process can infect all $n$ nodes), yet the stochastic diffusion infects only $O(\tau)$ nodes in expectation.

Proof. Take a cycle with $\ell$ nodes, say, $v_{0}, v_{1}, \ldots, v_{\ell-1}$. Now replace each $v_{i}$ by a clique $C_{i}$ on $\tau \geq 4$ nodes. Now for each $i$, connect vertices in clique $C_{i}$ to vertices in $C_{i+1}$ by a complete bipartite graph. Note that the total number of nodes $n$ equals $\tau \ell$. Also, one can verify that the toughness of this modified cycle is $\tau$, because the toughness of a simple cycle is 1 .

Assign a probability of 1 to edges inside each $C_{i}$, and probability $\frac{1}{2 \tau^{2}}$ to edges between the cliques. Note that for a pair of adjacent cliques, the probability that at least one edge between those cliques gets realized is less than $1 / 2$. Indeed, the probability that none of the edges between the two adjacent cliques get realized is more than $1 / 2$ :

$$
\left(1-1 /\left(2 \tau^{2}\right)\right)^{\tau} \geq 1-\frac{1}{2 \tau}>1 / 2,
$$

where we used the inequality $(1+x)^{r} \geq 1+r x$, for $x \geq-1$ and $r \in \mathbb{R} \backslash(0,1)$. In particular, the behavior of the stochastic diffusion on this network is essentially as it is on a cycle with edge-infection probability less than $1 / 2$, which was analyzed in the proof of Theorem 4.3; the only difference is that now each node on the cycle infects as well all the nodes in its clique. Thus, the stochastic diffusion infects at most $O(\tau)$ nodes in expectation. Finally, condition (5.2) trivially holds since $\tau \geq 4$, and so each vertex has a probability mass of at least 3 incidents on it, while $1 / \tau \leq 1$.

\section{CONCLUSIONS AND FUTURE WORK}

In this work we initiated the study of planned infection strategies, which can decide intelligently which nodes to infect next in order to maximize their spread, while obeying first-order constraints in order not to get discovered. We have demonstrated that a well-planned infection can substantially increase the number of nodes infected with respect to the standard Independent Cascades infection strategy. We designed necessary and sufficient conditions to understand when this is possible. Based on novel connection to the network toughness, we characterize networks where existence of planned strategies that are pandemic (infect all nodes) is guaranteed, as well as efficiently computable.

Our results have focused on first-order constraints: keeping the traffic involving any set $X$ lower than the ceiling of its expected value:

$$
\left\lceil\sum_{u \in X} \sum_{v:(u, v) \in E} p(u, v)\right\rceil .
$$

An interesting future direction is to consider flow constraints in which the number of infections caused by any set $X$ is at most the flow leaving $X$, namely,

$$
\left\lceil\sum_{u \in X} \sum_{v \notin X:(u, v) \in E} p(u, v)\right\rceil .
$$


Moreover, it would be interesting to consider directed graphs as well.

Another interesting avenue for further exploration is to analyze the effectiveness of vaccination strategies designed for controlling stochastic epidemics in limiting the spreading of a planned epidemic. In particular, one could consider immunization strategies such as immunizing high-degree nodes, or acquaintance immunization based on the immunization of a small fraction of random neighbors of randomly selected nodes. Such strategies are known to be effective at controlling stochastic epidemics [7,9] but might be ineffective containing the first-order constrained planned infections. To demonstrate this, let us go back to the example of the introduction, namely a path connected at one of its ends to the root of a two-level binary tree (see Figure 1). Consider vaccinating the node with the highest weighted degree, which, in this example, is the root of the tree. Vaccinating the root of the tree will not help contain a well-planned infection, which would still infect $n-O(1)$ nodes (the path nodes). Yet, vaccinating the middle node of the path is much better for containment-yielding at most $n / 2+O(1)$ infected nodes by any planned infection strategy. It is, therefore, interesting to understand and develop vaccination schemes that aim to minimize the number of infections against all planned strategies.

\section{ACKNOWLEDGMENTS}

We would like to thank the anonymous reviewers of Internet Mathematics and WAW' 13 for their helpful comments and suggestions.

\section{FUNDING}

Moez Draief was supported by QNRF grant NPRP-09-1150-2-448. Sanjeev Khanna was supported in part by the National Science Foundation grants CCF-1116961 and IIS1447470.

\section{APPENDIX:}

Lemma A.1. Let $r \geq 3$ be an integer divisible by 3. In Hamiltonian graphs with maximum degree $r$, the longest-path problem is not constant factor approximable, unless $\boldsymbol{P}=\mathrm{NP}$.

Proof. Consider a 3-regular Hamiltonian graph $G$. Construct a graph $H$ by simultaneously replacing each edge $(u, v)$ in $G$ by a clique $C_{(u, v)}$ on $r / 3+1$ nodes. First note that in $H$ the maximum degree is $r$ : each node that also appeared in $G$ has now degree $3 r / 3=r$. Now consider a node in the clique that is new (didn't appear in $G$ ). Its degree is $r / 3$ (one less the size of the clique). Now clearly a path of length $\ell$ translates to a path of length $\ell r / 3$ in $H$ by traversing the whole clique of $(u, v)$ for every edge $(u, v)$ in that path. And vice versa: a path in $H$ can always be extended in polynomial time to cover full cliques: if just part of a clique $C_{(u, v)}$ is covered that one can extend the path first to cover all of it. So assume without loss of generality that the path length $\ell^{\prime}$ is divisible by $r / 3$. Now one can recover a path in $G$ of length $\ell^{\prime} /(r / 3)$ by replacing each clique $C_{(u, v)}$ in the path by its original edge $(u, v)$ in $G$. In 3-regular Hamiltonian graphs, the longest-path problem is not constant factor approximable, unless $\mathbf{P}=\mathbf{N P}$ [6]. The lemma now follows immediately. 


\section{REFERENCES}

[1] R. Albert, H. Jeong, and A. L. Barabasi. "Error and Attack Tolerance of Complex Networks." Nature 406:6794 (2000), 378-382.

[2] N. Alon. "A Note on Network Reliability." In Discrete Probability and Algorithms, pp. 11-14. Springer, 1995.

[3] J. Balthrop, S. Forrest, M. E. J. Newman, and M. M. Williamson. "Technological Networks and the Spread of Computer Viruses." Science 304:5670 (2004), 527-529.

[4] D. Bauer, H. Broersma, and E. F. Schmeichel. "Toughness in Graphs - a Survey." Graphs and Combinatorics 22:1 (2006), 1-35.

[5] D. Bauer, H. Broersma, and H. J. Veldman. "Not Every 2-Tough Graph is Hamiltonian." Discrete Applied Mathematics 99:1-3 (2000), 317-321.

[6] C. Bazgan, M. Santha, and Z. Tuza. "On the Approximation of Finding A(nother) Hamiltonian Cycle in Cubic Hamiltonian Graphs." J. Algorithms 31:1 (1999), 249-268.

[7] T. Britton, S. Janson, and A. Martin-Löf. "Graphs with Specified Degree Distributions, Simple Epidemics, and Local Vaccination Strategies." Advances in Applied Probability 39:4 (2007), 922-948.

[8] Z. Chen, C. Chen, and C. Ji. "Understanding Localized-Scanning Worms." In IPCCC, pp. 186-193. IEEE, 2007.

[9] R. Cohen, S. Havlin, and D. Ben-Avraham. "Efficiency Immunization Strategies for Computer Networks and Populations." Physical Review Letters 91:24 (2003), 247901-1-247901-4.

[10] P. Crucitti, V. Latora, M. Marchiori, and A. Rapisarda. "Efficiency of Scale-Free Networks: Error and Attack Tolerance." Physica A 320:642 (2003), 622-642.

[11] D. A. Easley and J. M. Kleinberg. Networks, Crowds, and Markets - Reasoning About a Highly Connected World. New York, NY: Cambridge University Press, 2010.

[12] M. Fürer and B. Raghavachari. "Approximating the Minimum-Degree Steiner Tree to Within One of Optimal." J. Algorithms 17:3 (1994), 409-423.

[13] D. R. Karger, R. Motwani, and G. D. S. Ramkumar. "On Approximating the Longest Path in a Graph.” Algorithmica 18:1 (1997), 82-98.

[14] D. Kempe, J. M. Kleinberg, and É. Tardos. "Maximizing the Spread of Influence Through a Social Network." In Knowledge Discovery and Data Mining (KDD), pp. 137-146. New York, NY: ACM, 2003.

[15] M. M. Matthews and D. P. Sumner. "Hamiltonian Results in $\mathrm{K}_{1,3}$-Free Graphs." Journal of Graph Theory 8:1 (1984), 139-146.

[16] K. Ozeki and T. Yamashita. "Spanning Trees: A Survey." Graphs and Combinatorics 27:1 (2011), 1-26.

[17] G. Serazzi and S. Zanero. "Computer Virus Propagation Models." In MASCOTS Tutorials, pp. 26-50. IEEE/ACM, 2003.

[18] N. Weaver, V. Paxson, S. Staniford, and R. Cunningham. "A Taxonomy of Computer Worms." In WORM, pp. 11-18. ACM, 2003.

[19] S. Win. "On a Connection Between the Existence of $k$-Trees and the Toughness of a Graph." Graphs and Combinatorics 5:1 (1989), 201-205.

[20] G. Yan, G. Chen, S. Eidenbenz, and N. Li. "Malware Propagation in Online Social Networks: Nature, Dynamics, and Defense Implications.” In ASIACCS, pp. 196-206. New York: ACM, 2011.

[21] C. C. Zou, D. F. Towsley, and W. Gong. "Email Worms Modeling and Defense." In ICCCN, pp. 409-414. ICCCN, 2004.

[22] C. C. Zou, D. F. Towsley, and W. Gong. "On the Performance of Internet Worm Scanning Strategies." Perform. Eval. 63:7 (2006), 700-723.

[23] C. C. Zou, D. F. Towsley, W. Gong, and S. Cai. "Routing Worm: A Fast, Selective Attack Worm Based on IP Address Information.” In PADS, pp. 199-206. New York, NY: ACM, 2005. 